\title{
A new waist-to-height ratio predicts abdominal adiposity in adults.
}

\author{
NEVILL, A.M., STEWART, A.D., OLDS, T. and DUNCAN, M.J.
}

2020

This is an Accepted Manuscript of an article published by Taylor \& Francis in Research in Sports Medicine on 25/7/2018, available online: http://www.tandfonline.com/10.1080/15438627.2018.1502183. 
A new waist-to-height ratio predicts abdominal adiposity in adults

Alan M. Nevill ${ }^{1}$, Arthur D. Stewart ${ }^{2}$, Tim Olds ${ }^{3}$ and Michael J. Duncan ${ }^{4}$

1. Faculty of Education, Health and Wellbeing, University of Wolverhampton, Walsall, UK.

2. School of Health Sciences, Robert Gordon University, Aberdeen, UK.

3. School of Health Sciences, The University of South Australia, South Australia, Australia.

4. Faculty of Health and Life Sciences, Coventry University, UK.

Running head: Weight-status indices and waist adiposity

$\square$ Address for Correspondence:

Professor Alan M. Nevill,

University of Wolverhampton

Faculty of Education, Health and Wellbeing

Walsall Campus

Gorway Road

Walsall, WS1 3BD

Tel: +44 (0)1902322838

Fax: +44 (0)1902 322894

Email: a.m.nevill@wlv.ac.uk

\section{Acknowledgements}

The authors would like to thank Dr Richard Burton, University of Glasgow for advice on modelling SSK and all the participants that gave permission to use their data in these analyses. We received no funding (e.g. grants etc.) to conduct this work. 
A new waist-to-height ratio predicts abdominal adiposity in adults

\section{Abstract}

Our aim was to identify the best anthropometric index associated with waist adiposity. The six weightstatus indices included body mass index (BMI), waist-to-hip ratio (WHR), waist-to-height ratio (WHTR), and a new waist-by-height ${ }^{0.5}$ ratio (WHT.5R). The association between three waist skinfolds and the six anthropometric indices was conducted using ANCOVA, MANCOVA, allometric modelling and nonlinear regression. The strongest predictors of waist adiposity were $\left(1^{\text {st }}\right)$ WHT.5R, $\left(2^{\text {nd }}\right)$ WHTR, $\left(3^{\text {rd }}\right)$ waist circumference $(\mathrm{WC}),\left(4^{\text {th }}\right) \mathrm{BMI},\left(5^{\text {th }}\right) \mathrm{WHR}$, and lastly $\left(6^{\text {th }}\right)$ a body shape index $\mathrm{ABSI}=\mathrm{WC} /\left(\mathrm{BMI} \mathrm{I}^{2 / 3}\right.$ ${ }^{*}$ height ${ }^{1 / 2}$ ). The allometric and non-linear regression analyses identified the optimal waist-to-height ratio associated with waist adiposity to be (waist ${ }^{*}$ height ${ }^{-0.6}$ ). The $95 \%$ confidence intervals of the height exponents encompassed -0.5 but excluded -1.0 assumed by WHTR. Assuming that excess waist adiposity is an important cardiovascular risk factor, we recommend that the new WHT.5R be used to advise people how to maintain a "healthy" weight.

Keywords: Allometric Models; Waist-to-Height ratios; Abdominal Skinfolds; Healthy Weight 


\section{Introduction}

Clinicians and health professionals are always seeking to find a simple weight-status index associated with cardio-metabolic risk that they can use to advise either their patients or members of the public how to maintain a "healthy" weight. The World Health Organization considers body mass index (BMI =weight $(\mathrm{kg}) /$ height $^{2}\left(\mathrm{~m}^{2}\right)$, to be such an index, with the advice that having a BMI within the range 18.5 to $24.9 \mathrm{~kg} \cdot \mathrm{m}^{-2}$ was normal, $25-29.9 \mathrm{~kg} \cdot \mathrm{m}^{-2}$ was overweight and $30 \mathrm{~kg} \cdot \mathrm{m}^{-2}$ and above was obese (WHO, 2000). Despite its widespread use, BMI is often criticized for failing to assess fatness, in particular the fat centralization on the abdomen, which is recognized as harmful to health. Although visceral and intra abdominal fatness are more precisely determined using computerized tomography, magnetic resonance imagining and dual x-ray absorptiometry (DEXA) (Roriz, et al., 2014), anthropometric measures provide a time efficient and economical means of estimating central adiposity and weight status that are widely used in public health practice. As a consequence, a range of alternative anthropometric indices which encompass some abdominal girth assessment have sought to overcome the limitations of BMI, including waist circumference (WC), waist to height ratio (WHTR) and a Body Shape Index (ABSI) as defined by Krakauer and Krakauer (2012). Excess intraabdominal fat is associated with greater risk of obesity-related morbidity than is overall adiposity (Ho, et al., 2001; Visscher, Kromhout, \& Seidell, 2002) and, as a consequence, waist circumference (WC) has historically been shown to be the best simple measure of both intra-abdominal fat mass and total fat (Han, McNeill, \& Seidell, 1997; Lemieux, Prud'homme, Bouchard, Tremblay, \& Desprs 1996). A recent study by Nevill, Duncan, Lahart, \& Sandercock (2017) suggests that a better index for adiposity that also demonstrates a stronger association with cardio-metabolic risk is a new waist-to-height ratio (WHT.5R) obtained by dividing WC by height ${ }^{0.5}$. The index was derived, based on the allometric power law $\mathrm{WC}=\mathrm{a} \cdot \mathrm{HT}^{\mathrm{b}}$, in order to obtain a waist circumference index that is independent of height $(\mathrm{HT})$. The fitted HT exponent was $b=0.528$ ( $\mathrm{SEE}=0.04$ ), suggesting that the simple body-shape or weight-status index for WC to be independent of stature or height $(\mathrm{HT})$ should be $\mathrm{WC} . \mathrm{HT}^{-0.5}$. A new ratio was given the acronym WHT.5R. Subsequent research by Swainson, Batterham, Tsakirides, Rutherford, \& Hind (2017) recently reported that WHT.5R, alongside WHTR, was the best predictor of visceral adipose tissue, derived by DEXA in a sample of 81 adults. However, these results should be treated with some caution given the relatively small sample size and the fact that the authors failed to control for the confounding effect of age. 
Nevill et al. (2017) went on to demonstrate that when comparing the associations of the six adiposity indices (including BMI) with a measure of cardio-metabolic risk, the new index WHT.5R was found to have the strongest relationship of all six indices. In their study, Nevill, et al (2016) used a measure of cardio-metabolic risk that was derived from combining: Triglycerides + average blood pressure $(($ diastolic + systolic $) / 2)+$ glucose + HDL $\left.\left({ }^{*}-1\right)\right)$. Although WHT.5R was found to have the strongest association with cardio-metabolic risk (rank=1), the second and third strongest ranked indices were the waist-to-height ratio (WHTR) and absolute WC respectively, leaving BMI ranked only fourth out of the six indices (Nevill, et al., 2017). Nevill, et al (2017) concluded that WHT.5R was the best anthropometric index of cardio-metabolic risk compared to other commonly-used anthropometric indices of weight status. Importantly, the findings of the aforementioned study suggest that weightstatus indices that can detect excess subcutaneous central obesity are more likely to detect cardiometabolic risk. Waist circumference may be a more sensitive measure to detect changes in cardiometabolic health but normalizing for height to the power of 0.5 may be a more suitable anthropometric index as height ${ }^{0.5}$ is unaffected by changes in adiposity unlike measures such as hip girth that was used as the denominator in WHR and BMI, used as a denominator in ABSI. However, the aforementioned study by Nevill, et al (2017) did not examine the association between WHT.5R and any measure of adiposity and much remains to be discovered regarding the relationship of directly measured subcutaneous central obesity assessed using skinfolds to the anthropometric indices available to assess health. The current study addresses this issue. Determining the association between measures of weight status using surface anthropometry with directly measured adiposity is a necessary step in establishing the utility and criterion validity of such measures as proxies for adiposity. Hence the purpose of the current study was to examine this association and identify the best weight-status index associated with subcutaneous central obesity, the latter being assessed by three measures of skinfolds that represent adiposity around the waist region, i.e., the iliac crest, supraspinale, abdominal skinfolds. We hypothesise that the new waist-to-height ratio WHT.5R will be the best anthropometric ratio to reflect subcutaneous central obesity. 


\section{METHODS}

\section{Participants}

All participants $(n=478)$ were adults (18 years or over), and included untrained controls, and athletes in a variety of sports competing at club, regional and national level. They were measured either by level 3 and 4 anthropometrists accredited by the International Society for the Advancement of Kinanthropometry (ISAK), at Universities in Aberdeen, UK, and South Australia. All measurements were made subject to informed consent and in accordance with the ethical requirements of the local institutions. Participants were measured for height, mass, waist and hip girth, and skinfolds at the iliac crest, supraspinale and abdominal sites according to standard procedures (Stewart, Marfell-Jones, Olds, \& de Ridder, 2011). The physical characteristics (mean \pm SD) of the participants, by training status (athlete vs control) and sex, are given in Table 1. Healthy male and female participants were classified according to training status, with athletes taking part in a variety of sports including running, orienteering, long jumping, power lifting and field games such as football and hockey.

Table 1 near here -

\section{Anthropometric indices of weight status}

The anthropometric indices of weight status were calculated as follows: Body mass index (BMI) was calculated as weight $/$ height $^{2}$ with weight assessed to $0.1 \mathrm{~kg}$ and stature to $0.001 \mathrm{~m}$. Waist-to-hip ratio (WHR) was calculated by dividing waist circumference (WC) by hip circumference (HC), and waist-toheight ratio WHTR, by dividing WC by height. Both girths were assessed to $0.1 \mathrm{~cm}$. A body shape index $(\mathrm{ABSI})$ was calculated using formula: $\mathrm{ABSI}=\mathrm{WC} /\left(\mathrm{BMI}^{2 / 3}\right.$ * height $\left.{ }^{1 / 2}\right)($ Krakauer and Krakauer, 2012). Finally, a new waist-to-height ratio (WHT.5R), independent of height, was calculated by dividing WC by height ${ }^{0.5}$ (see Nevill, et al., 2017). Note that all calculations of anthropometric indices involving height and WC are in recorded in metres $(m)$.

\section{Procedures}

Three skinfold sites in close proximity to the measured waist circumference were taken as our measure of subcutaneous adiposity. The three sites and their respective skinfold orientations were 1) the iliac crest: a near-horizontal fold superior to the iliac crest (defined where a vertical line from the 
axilla meets the ilium); 2) supraspinale: an oblique fold at approximately $45^{\circ}$ at the intersection where a line from the anterior superior iliac spine to the anterior axillary fold meets a horizontal line from iliac crest; and 3) abdominal: a vertical fold $5 \mathrm{~cm}$ lateral to the omphalion (the mid-point of the navel). The skinfolds were measured using Harpenden calipers (British Indicators, Luton, UK) to $0.1 \mathrm{~mm}$ at each site on the right side of the body, according to standard procedures (Stewart et al., 2011).

\section{Statistical Methods}

Intially, Peason's product-moment correlations were used to explore the association between the different variables (unadjusted for age and sex). To explore the strength of the association between the three waist skinfold measures (either individually or collectively) and six anthropometric indicators of weight status (BMI, WC, WHR, WHTR, ABSI and WHT.5R) now controlling for age and sex, we conducted five methods of analysis. The first two methods involve considering the three skinfold measures collectively, either (1) by summing the three skinfolds (SSF) and using ANCOVA to identify the best weight-status index as a predictor/covariate having controlled for age, sex and athletic status (athlete vs. control), or (2) by conducting a MANCOVA (adopting all three skinfold variables as the multivariate dependent variable), once again incorporating the six anthropometric indicators of weight status as separate covariates, and controlling for age, sex and athletic status (athlete vs. control). The third method of analyses (3) used separate univariate ANCOVAs to explore each skinfold variable separately, i.e., incorporating each of the six anthropometric indicators as separate covariates, once again controlling for age, sex and athletic status (athlete vs. control). The fourth method of analysis (4), adopted a proportional allometric model (see Nevill, et al., 2006), to identify which waist-to-height ratio is best able to describe the sum-of-waist skinfolds (SSF). The proposed proportional allometric model was,

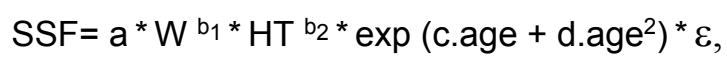

where $a$ is the scaling constant, allowed to vary between sex and athletic status, and $b_{1}$ and $b_{2}$ are the waist (W) and stature (HT) scaling exponents respectively. Note that age was incorporated into the models as a quadratic polynomial (using both age and $a^{2} \mathrm{e}^{2}$ terms) to accommodate the likelihood that the sum of skinfolds may decline during adolescence to reach a minimum sometime in early adulthood and increase thereafter. The model (1) can be linearized with a log-transformation, and multiple regression/ANCOVA can then be used to estimate unknown parameters, including the waist (W) and 
stature $(\mathrm{HT})$ exponents. Note that the multiplicative error ratio ' $\varepsilon$ ' assumes that the error will increase in proportion to body size (see Figure 1), a characteristic in data known as heteroscedasticity that can be controlled by taking logarithms. Categorical or group differences within the population, e.g. sex and athletic status can be explored by allowing the constant intercept parameter ' $a$ ' in (1) to vary for each group (by introducing them as fixed factors and their interactions within the ANCOVA).

Finally, recognising that the allometric model (1) forces the association between SSK and the waist-by-height ratio through the origin $(Y=0, X=0)$, we fitted an alternative non-linear model that allows a non-zero constant intercept $\mathrm{W}_{0}$ to exist as SSK tends towards zero.

$$
\mathrm{SSF}+\mathrm{W}_{0}=\mathrm{a}^{*} \mathrm{~W}^{*} \mathrm{HT}^{\mathrm{b}}{ }^{*} \exp \left(\mathrm{c} \cdot \text { age }+ \text { d.age }{ }^{2}\right),
$$

The model (2) was rearranged as follows

$$
\mathrm{SSF}=\mathrm{a}^{*} \mathrm{~W}^{*} \mathrm{H} \mathrm{T}^{\mathrm{b}}{ }^{*} \exp \left(\mathrm{c} \cdot \text { age }+\mathrm{d} \cdot \mathrm{age}^{2}\right)-\mathrm{W}_{0},
$$

and fitted using the non-linear least-squares regression routine provided in SPSS.

For all the above analyses, the significance level was set at $P<0.05$.

\section{RESULTS}

A strong association between the sum-of-waist skinfolds (iliac crest, supraspinale, abdominal skinfolds) and waist circumference, can be seen in Figure 1. The Pearson's correlations between the sum-of-waist skinfolds and waist circumference were, for male controls $(r=0.67 ; P<0.001)$, female controls $(r=0.64 ; P<0.001)$, male athletes $(r=0.64 ; P<0.001)$, female athletes $(r=0.67 ; P<0.001)$ and for all four groups combined $(r=0.42 ; P<0.001)$,

Figure 1 about here -

A two-way ANOVA reveals significant differences in the sum-of-waist skinfolds between athletic status (controls mean $40.8 \mathrm{~mm}(\mathrm{SE}=1.3)$ vs. athletes mean $35.6 \mathrm{~mm}(\mathrm{SE}=1.2) ; \mathrm{P}=0.004)$ but no difference between sex nor a sex-by-athletic status interaction (both $\mathrm{P}>0.05$ ). When the four groups were analysed using a one-way ANOVA, the rank order in sum-of-waist skinfolds was as follows (male controls $=43.4 \mathrm{~mm}$ female controls $=38.1 \mathrm{~mm}$, female athletes $=35.8 \mathrm{~mm}$, and male athletes $=35.3 \mathrm{~mm}$; $\mathrm{P}=0.004)$. 
In order to assess the extent to which the six anthropometric indices made to the above two-way ANOVA, each anthropometric index was incorporated as covariates separately. The resulting covariate $\mathrm{F}$ ratios from the six ANCOVA analyses are given in Table $2 \mathrm{a}$, having controlled for age (incorporated as a quadratic), athletic status and sex.

The MANCOVA analyses adopting the three skinfold measures as the multivariate dependent variable identified the following Wilk's lambda and F ratio contributions for the six anthropometric covariates having controlled for age (incorporated as a quadratic), athletic status and sex. The results are given in Table 2b. In all six MANCOVA analyses, the effects of the quadratic terms in age, athletic status sex were significant $(P<0.001)$. Note that the Wilks lambda ranges from 0 to 1 and the lower the Wilks lambda, the stronger the relationship. This was confirmed by the larger $\mathrm{F}$ ratios.

Table 2 about here -

Further evidence (using the covariate $F$ ratios) of the strength of association between subcutaneous adiposity (adopting the three separate skinfolds of iliac-crest, supraspinale, abdominal skinfolds as separate dependent variables) and the six weight-status anthropometric covariates, having controlled for age (as a quadratic), athletic status and sex in the 18 univariate ANOVAs, are given in Table 3. As before, in all 18 ANOVAs, the effects of age, athletic status and sex were significant $(P<0.001)$.

Table 3 about here -

Using the proportional allometric model (1), the ANCOVA of log-transformed sum-of-waist skinfolds $\mathrm{Ln}(\mathrm{SSF})$ resulted in the following results and fitted parameters (Table 4).

Table 4 about here -

The waist (3.751) and height (-2.340) exponents associated with the log-transformed sum-of-waist skinfolds in the above model have opposite signs that can be rearranged and expressed as a waist-toheight ratio within a power function relationship as follows:

$$
\text { Waist }^{3.751} \cdot \text { height }^{-2.34}=\left(\text { waist } \cdot \text { height }^{-0.624}\right)^{3.751} \text {, }
$$


since height ${ }^{-2.34}=\left(\text { height }^{-0.624}\right)^{3.751}$. The $95 \%$ confidence interval $(\mathrm{Cl})$ for the rearranged/rescaled height exponent -0.624 is $(-0.826$ to -0.440$)$. Note that the quadratic in age is " $U$ " shaped and reaches a minimum at age $=-(-0.03) / 2^{*}(.0004)=37.5$ years (using elementary differential calculus).

Finally, the results and fitted parameters, obtained from fitting the non-linear least-squares regression model (equation 3) using the SPSS non-linear least-squares regression algorithm, are given in Table 5.

Table 5 about here -

Once again, the stature exponent $b=-0.641$ (SE=0.092) had a 95\% confidence interval $(\mathrm{Cl})$ from 0.822 to -0.460 , very similar to that estimated and reported using the allometric model (1) in Table 4 . Note that the quadratic in age is " $U$ " shaped and reaches a minimum at age $=-(-0.007) / 2^{*}(8.77 \mathrm{E}$ 06) $=39.9$ years (again using elementary differential calculus).

\section{DISCUSSION}

The current study examined the association between commonly-used surface anthropometric surrogates for subcutaneous central obesity and subsequently determined a hierarchy of preference of the indices using a variety of statistical approaches. This is the first study to present such data and, as such, adds new insight into the literature on the topic of which anthropometric index is the best proxy for centralized adiposity. Such information directly relates to health, is of pivotal value for accurate translation into public health practice, and could be used to refine anthropometric proxies for adiposity as used in healthcare policy worldwide. Understanding the strength of association between surface anthropometric measures and subcutaneous central obesity in healthy, non-obese, adults is equally as important as determining this association in obese individuals for two reasons. First, it is commonly overlooked that the antecedents of obesity might be present in individuals whose weight has been historically considered to be in the healthy range. Secondly, the cut-offs of reference ranges and categorization has the potential to mislead, because such a 'cliff edge' phenomenon has no equivalent physiological basis. To clarify, the commonly used BMI cut point of $30 \mathrm{~kg} / \mathrm{m}^{2}$ to classify an individual 
as obese does not have a physiological basis (See Nevill and Holder, 1995). The validity of BMI is based on the assumption that as BMI increases so does adiposity but classifying based on $30 \mathrm{~kg} / \mathrm{m}^{2}$ has no biological validity (Nevill and Holder, 1995) and instead appears to have arisen from the need for a convenient measure of weight status for statistical analysis and classification of individuals at a population level. The current study demonstrates the erroneous nature of such a 'cliff edge' approach (i.e., classification of individuals as obese/non-obese based on BMI) as erroneous for the participant group examined as, for example, athletically trained adults may be classified as obese, based on BMI, but in reality, may be lean but muscular. The participants in the current study comprised untrained and athletically trained adults. Determining associations between surface anthropometry and subcutaneous central obesity in such a population is useful as it allows a better understanding of which anthropometric measure might have best clinical relevance in a sample that are yet to present with obesity. Such information is key for preventive purposes and population-based screening of weight status before individuals become obese and recognizing that adiposity may have associated metabolic abnormality in the absence of obesity (Amato, Guarnotta, \& Giordano, 2013).

The results of the first three methods of analyses identified the strongest predictor of, or association with, subcutaneous central obesity (using the three waist skinfolds either collectively in Tables $2 \mathrm{a}$ and $2 \mathrm{~b}$ or separately in Table 3 ) was the waist-to-height ${ }^{0.5}$ ratio. Indeed, the strongest through to the weakest anthropometric covariate associated with subcutaneous central obesitywas found to be the following order, \#1 the waist-to-height ${ }^{0.5}$ ratio (WHT.5R), \#2 waist-to-height ratio (WHTR), \#3 absolute waist, \#4 BMI, \#5 waist-to-hip ratio (WHR), and finally \#6 ABSI, an order that remained consistent in all three types of analyses, see Tables $2 a, 2 b$, and 3 . The second and third best predictors of subcutaneous central obesity were waist-to-height ratio and absolute waist circumference, certainly better than either BMI, waist-to-hip ratio, or ABSI. This rank order is identical with that reported by Nevill et al. (2017) when reporting the associations between weight status indices and cardio-metabolic risk using an entirely independent population.

Further evidence from the current study that the WHT.5R index is the best (rank=1) indicator of subcutaneous central obesity compared with the WHTR (rank=2) comes for the fourth "allometric" and fifth "non-linear regression" analyses. These analyses identified the optimal waist-to-height ratio associated with the sum-of-waist skinfolds was (waist $\cdot$ height ${ }^{-0.624}$ ) and (waist $\cdot$ height ${ }^{-0.641}$ ) respectively (having controlled for differences in age, sex and athletic status). The empirically derived 
ratios are not too dissimilar to the WHT.5R since both $95 \%$ confidence intervals $(\mathrm{Cl})$ encompass the height exponent -0.5 but precludes -1.0 (i.e., that assumed for the waist-to-height ratio, WHTR).

Interestingly, both quadratics in age from Tables 4 and 5 suggest that, for the same waist-byheight ratio (approximately waist $\cdot$ height ${ }^{-0.6}$ ), the waist-skinfold adiposity reaches a minimum at age 37-40 years. This period broadly corresponding to an age when both fat-free mass and appendicular muscle mass appear to commence an age-related decline in healthy adults (Kyle et al., 2001). Furthermore, having controlled for age and body size, BOTH sex and athletic status were now significant, see Tables 4 and 5 . This further underscores the inappropriateness of using body weight alone to predict adiposity, because age-related muscle loss and fat gain may balance one another out (Spirduso, 1995, p 68). Although Ashwell, Gunn, \& Gibson (2012) concluded, having performed a systematic review and meta-analysis with data representing over 300,000 adults, that WHTR (rank $2^{\text {nd }}$ in the current study) was superior to both WC (ranked $3^{\text {rd }}$ ) and BMI (ranked $4^{\text {th }}$ ) for detecting cardiometabolic risk in both males and females, the WHTR suffers from the following drawback: Absolute or unadjusted waist circumference (WC) will clearly penalise the taller subject who might have a larger waist suggesting that their cardio-metabolic risk is greater than a shorter individual with the same waist circumference. However, by dividing waist by height, the waist-to-height ratio (WHTR) will "over-scale" an individual's waist circumference predicting a taller subject to have a lower cardio-metabolic risk than the shorter individual with the same waist circumference. The only index that will do neither overor under-estimate the subject's cardio-metabolic risk would appear to be the WHT.5R, found to be independent of height/stature. Height ${ }^{0.5}$ is unaffected by changes in adiposity whereas measures such as BMI (adopted as a denominator in the ABSI) and hip girth, (adopted as a denominator in WHR), are (Nevill, et al., 2017), i.e., by dividing WC by such body-size dimensions also known to be inflated by waist adiposity will lead to a dilution in the impact of the ratio to detect abdominal adiposity. Thus, normalizing or scaling waist girth for height ${ }^{0.5}$ is more suitable in providing a proxy for subcutaneous central obesity that accounts for, but is independent of, differences in body size. Such an assertion also agrees with conclusions recently made by Swainson et al (2017) based on their analysis of DEXA determined visceral adipose tissue. It would however be prudent for additional study to cross validate the methods presented in the current study.

Across the epidemiological literature, there has been justifiable criticism of some of the early indices to be adopted such as BMI and WHR including failing to represent adiposity, or being 
insensitive to tracking its changes (Ho, et al, 2001; Javed, et al., 2015; WHO, 2000). It is also important to note that the definitions of overweight and obesity commonly used in the literature and recommended by the WHO (2000) (BMI $\geq 25$ and $\geq 30 \mathrm{~kg} \cdot \mathrm{m}^{-2}$ respectively) were based on the relationship between BMI with morbidity and mortality outcomes (Dalton, et al., 2003). Nevertheless, BMI remains in widespread use to classify weight status in relation to body fat, yet crucially fails to take into account the composition of body mass (e.g., muscle vs fat) and nor does it account for the distribution of excess fat. WHR had previously been acknowledged as a clinically relevant method of identifying patients with excess abdominal fat, although fat accumulation in the hip area, or a large overhanging abdomen which reaches hip level may mean this ratio fails to alter with increasing fatness. Latterly WC has been identified as a more practical measure than WHR which is more strongly correlated with visceral fat than WHR (Camhi, et al., 2011; Pouliot, et al., 1994). However, viable alternatives to these have experienced considerable resistance, which may be due to development of reference ranges for different population groups. Our data not only demonstrate how poorly such trusted ratios perform, but point to a hitherto unexplained flaw in most of these, and elucidate an alternative which does not artificially penalise taller or shorter individuals. We however recognise that the current study does have some limitations. Notably the associations reported here were developed on a reativle lean population and only subcutaneous fat was considered.

The data presented in the present study add support to the findings of Nevill et al (2016) in regard to the utility of WHT.5R. When attempting to determine the superiority of simple anthropometric measures of weight status, assessing the association of that measure with various disease risk factors, as was demonstrated by Nevill et al (2017), is essential but forms only part of the answer. The present study establishes the validity of WHT.5R as a measure of subcutaneous central obesity that was superior to all other simple anthropometric indices of obesity. In this case, the present study demonstrates the criterion validity of WHT.5R. This should be considered a key novel finding of the present work. Finally, consideration needs to be given to the practicality and accuracy of measurement, particularly if widespread use in public health settings for health screening is intended. It should be noted that WHT.5R is no more difficult to measure than BMI or WC. WHT.5R therefore provides a measure which is more strongly related to cardio-metabolic risk, as demonstrated previously, is more strongly related to subcutaneous central obesity and is as easy to assess with high accuracy as any other simple anthropometric measure of weight status or obesity. 
Implementing the model has considerable application in clinical and health settings. This centres on the recognition that 1) overweight status may be the antecedent of obesity and 2) that athletic individuals with large frame size and considerable muscle development are misrepresented by generic models. Just as is the case in sarcopenic obesity, when fat gain is masked or balanced by muscle loss, a young athletic sample needs to be viewed as a special case. In this paper we have considered this effect by virtue of athlete-control as well as male-female comparison, and demonstrated that an athletic sample may exhibit subtle differences as compared to a non-athletic one. As the physical activity message continues be 'shouted from the rooftops' of public health and wellbeing organisations, this finding has implication and impact for the wider population.

In conclusion, the current study has identified that the new index WHT.5R was the strongest predictor of subcutaneous central obesity. Given that WHT.5R is independent of stature and is also the strongest predictor of cardio-metabolic risk (see Nevill, et al., 2017), we recommend that clinicians and health professionals should consider adopting the new waist-by-height ${ }^{0.5}$ ratio (WHT.5R) to advise either their patients or members of the public how to maintain a "healthy" weight. 


\section{References}

Amato, M. C., Guarnotta, V., Giordano, C. (2013). Body composition assessment for the definition of cardiometabolic risk. Journal of Endocrinological Investigation, 36, 537-543.

Ashwell, M., Gunn, P., \& Gibson, S. (2012). Waist to height Ratio is a Better Screening Tool than Waist Circumference and BMI for Adult Cardiometabolic Risk Factors: Systematic Review and Meta analysis. Obesity Reviews, 13, 275-286.

Camhi, S. M., Bray, G. A., Bouchard, C., Greenway, F. L., Johnson, W. D., Newton, R. L., Ravussin, E., Ryan, D. H., Smith, S. R., \& Katzmarzyk, P.T. (2011). The Relationship of Waist Circumference and BMI to Visceral, Subcutaneous, and Total Body Fat: Sex and Race Differences. Obesity, 19, 402-408.

Dalton, M., Cameron, A. J., Zimmet, P. Z., Shaw, J. E., Jolley, D., Dunstan, D. W., \& Welborn, T. A. (2003). Waist Circumference, Waist-hip Ratio and Body Mass Index and their Correlation with Cardiovascular Disease Risk Factors in Australian Adults. Journal of Internal Medicine, 254, 555563.

Han, T. S., McNeill, G., Seidell, J. C., \& Lean, M. (1997). Predicting Intra-Abdominal Fatness from Anthropometric Measures: The Influence of Stature. International Journal of Obesity \& Related Metabolic Disorders, 1997, 21.

Ho, S. C., Chen, Y. M., Woo, J., Leung, S., Lam, T. H., \& Janus, E. D. (2011). Association between Simple Anthropometric Indices and Cardiovascular Risk Factors. International Journal of Obesity, 25, 1689.

Javed, A., Jumean, M., Murad, M. H., Okorodudu, D., Kumar, S., Somers, V. K., Sochor, O., \& LopezJimenez, F. (2015). Diagnostic Performance of Body Mass Index to Identify Obesity as Defined by Body Adiposity in Children and Adolescents: A Systematic Review and Meta analysis. Pediatric Obesity, 10, 234-244.

Krakauer, N.Y., \& Krakauer, J. C. (2012). A New Body Shape Index Predicts Mortality Hazard Independently of Body Mass Index. PloS One, 7, e39504.

Kyle, U.G., Genton, L., Hans, D., Karsegard, L., Slosman, D.O. \& Pichard, C. (2001). Age-related differences in fat-free mass, skeletal muscle, body cell mass and fat mass between 18 and 94 years. European Journal of Clinical Nutrition, 55, 663-672.

Lemieux, S., Prud'homme, D., Bouchard, C., Tremblay, A., \& Desprs, J. (1996). A Single Threshold Value of Waist Girth Identifies Normal-Weight and Overweight Subjects with Excess Visceral Adipose Tissue. American Journal of Clinical Nutrition, 64, 685-693.

Nevill, A. M., Duncan, M. J., Lahart, I. M., \& Sandercock, G. R. (2017) Scaling Waist Girth for Differences in Body Size Reveals a New Improved Index Associated with Cardiometabolic Risk. Scandinavian Journal of Medicine and Science in Sports, e-pub ahead of print.

Nevill, A. M., Stewart, A. D., Olds, T., \& Holder, R. (2006). Relationship between Adiposity and Body Size Reveals Limitations of BMI. American Journal of Physical Anthropology, 129, 151-156.

Nevill, A. M., Holder, R. L. (1995). Body mass index: a measure of fatness or leanness? British Journal of Nutrition, 73, 507-516.

Pouliot, M., Desprs, J., Lemieux, S., Moorjani, S., Bouchard, C., Tremblay, A., Nadeau, A., \& Lupien, P.J. (1994). Waist Circumference and Abdominal Sagittal Diameter: Best Simple Anthropometric Indexes of Abdominal Visceral Adipose Tissue Accumulation and Related Cardiovascular Risk in Men and Women. American Journal of Cardiology, 73, 460-468.

Roriz, A. K. C., Passos, L. C. S., de Oliveira, C. C., Eickemberg, M., Moreira, P. d.A. , Sampaio, L. R. (2014), Evaluation of the Accuracy of Anthropometric Clinical Indicators of Visceral Fat in Adults and Elderly. PLoS ONE,9, e103499. 
Spurduso. W. W. (1995). Physical dimensions of aging. Champaign, IL: Human Kinetics, p 68.

Stewart, A., Marfell-Jones, M., Olds, T., \& Ridder, d.H. (2011). International Society for Advancement of Kinanthropometry. International Standards for Anthropometric Assessment. Lower Hutt, New Zealand: International Society for the Advancement of Kinanthropometry.

Swainson, M. G., Batterham, A. M., Tsakirides, C., Rutherford, Z. H., Hind, K. (2017). Prediction of whole-body fat percentage and visceral adipose tissue mass from five anthropometric variables. PLoS ONE, 12, e0177175

Visscher, T., Kromhout, D., \& Seidell, J.C. (2002). Long-Term and Recent Time Trends in the Prevalence of Obesity among Dutch Men and Women. International Journal of Obesity, 26, 1218.

World Health Organization (2000). Obesity: Preventing and Managing the Global Epidemic. Geneva, World Health Organization . 


\section{FIGURE CAPTIONS}

Figure 1. The association between the sum-of-waist skinfolds (iliac crest, supraspinale, abdominal skinfolds) and waist circumference by sex and group (athletes versus controls). 


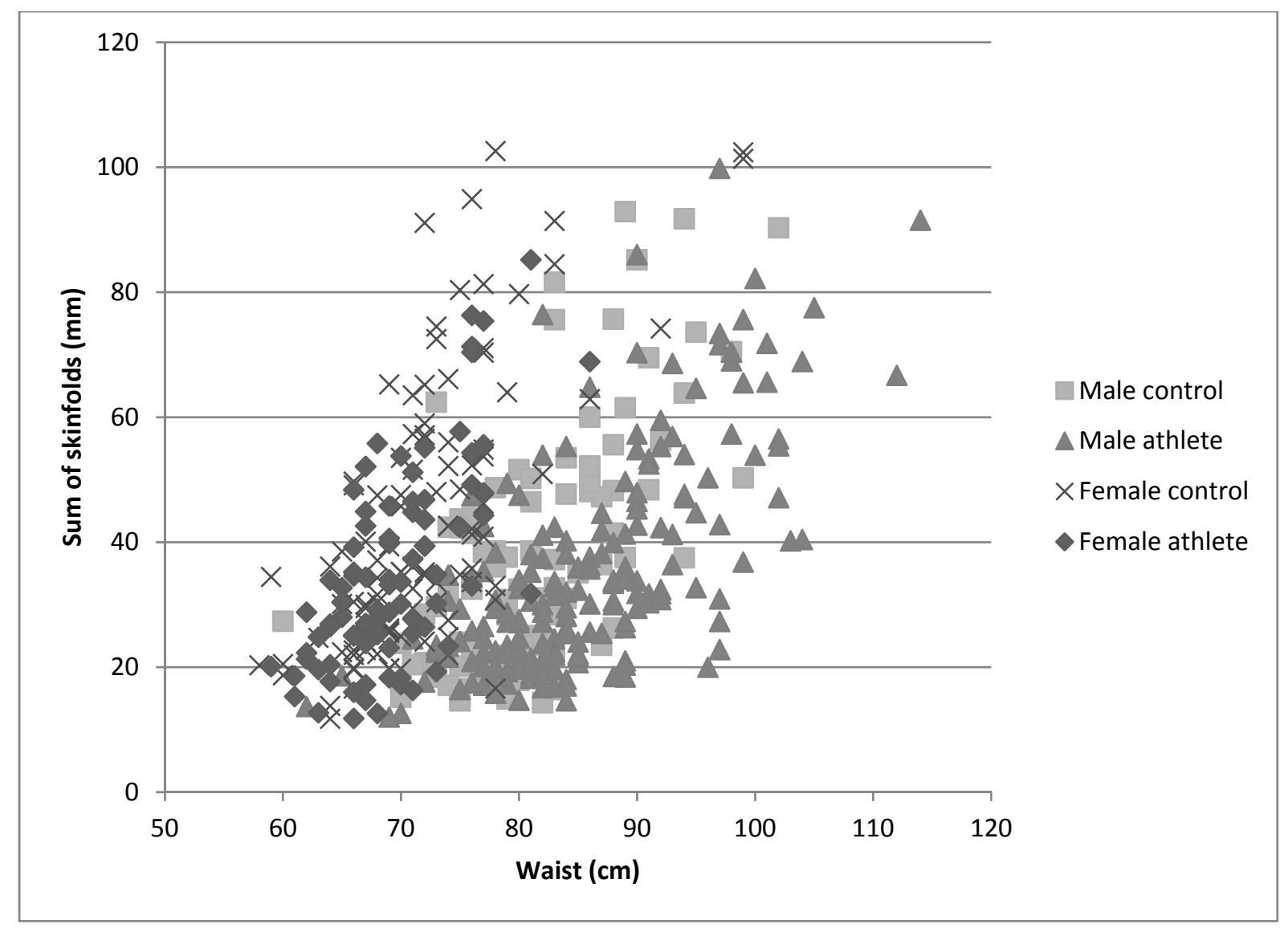


Table 1. Physical characteristics of participants

\begin{tabular}{lllllll} 
Female & & age $(\mathrm{y})$ & mass $(\mathrm{kg})$ & stature $(\mathrm{cm})$ & BMI $\left(\mathrm{kg} \cdot \mathrm{m}^{-2}\right)$ & waist girth $(\mathrm{cm})$ \\
\hline Control $(\mathrm{n}=104)$ & Mean & 30.2 & 61.7 & 166.0 & 22.4 & 71.8 \\
& SD & 8.8 & 8.7 & 6.1 & 2.6 & 6.8 \\
Athletes (n=87) & Mean & 27.9 & 60.5 & 168.4 & 21.3 & 69.3 \\
& SD & 9.0 & 9.0 & 8.6 & 2.1 & 5.0 \\
Total $(\mathrm{n}=191)$ & Mean & 29.1 & 61.2 & 167.1 & 21.9 & 70.7 \\
& SD & 8.9 & 8.8 & 7.5 & 2.5 & 6.1 \\
Male & & & & & & \\
\hline Control $(\mathrm{n}=95)$ & Mean & 30.0 & 75.2 & 178.5 & 23.6 & 81.2 \\
& SD & 9.6 & 9.2 & 8.2 & 2.3 & 7.2 \\
Athletes (n=192) & Mean & 26.8 & 86.5 & 182.7 & 25.8 & 85.6 \\
& SD & 7.6 & 14.6 & 9.0 & 3.5 & 8.7 \\
Total $(\mathrm{n}=287)$ & Mean & 27.9 & 82.7 & 181.3 & 25.1 & 84.2 \\
& SD & 8.4 & 14.1 & 9.0 & 3.3 & 8.5 \\
\hline
\end{tabular}

$\mathrm{SD}=$ Standard Deviation 
Table 2. The contributions for the six anthropometric weight-status covariates to a) the univariate ANCOVA (using the sum of skinfolds as the dependent variable), and b) MANCOVA (using the iliac-crest, supraspinale, abdominal skinfolds as the three dependent variables) having controlled for age, athletic status and sex. Rank order with 1=best fit through to the $6=$ worst fit

\begin{tabular}{llllll}
$\begin{array}{l}\text { 2a. Univariate ANCOVA using } \\
\text { Sum-of-skinfolds as dependent } \\
\text { variable. }\end{array}$ & $\begin{array}{l}\text { Rank } \\
\text { order }\end{array}$ & $\begin{array}{l}\text { 2b.MANCOVA with three } \\
\text { skinfold as dependent } \\
\text { variables }\end{array}$ & $\begin{array}{l}\text { Rank } \\
\text { order }\end{array}$ \\
\hline $\begin{array}{l}\text { Anthropometric } \\
\text { covariate variables }\end{array}$ & F1,468 & & $\begin{array}{l}\text { Wilks' } \\
\text { Lambda }\end{array}$ & F $_{3,466}$ & \\
BMI & 244.176 & 4 & 0.635 & 89.557 & 4 \\
Waist & 291.694 & 3 & 0.608 & 100.21 & 3 \\
WHR & 58.009 & 5 & 0.888 & 19.547 & 5 \\
WHTR & 337.543 & 2 & 0.569 & 117.67 & 2 \\
ABSI & 20.224 & 6 & 0.953 & 7.730 & 6 \\
WHT.5R & 353.602 & 1 & 0.559 & 122.51 & 1 \\
\hline
\end{tabular}


Table 3. The contributions (F ratios) for the six anthropometric weight-status covariates associated with the three separate skinfolds dependent variables (the iliac-crest, supraspinale, abdominal skinfolds) having controlled for age (as a quadratic), athletic status and sex.

Skinfolds

\begin{tabular}{lllll}
\hline $\begin{array}{l}\text { Anthropometr } \\
\text { ic }\end{array}$ & Iliac-crest & Supraspinale & Abdominal & Rank order \\
\cline { 2 - 5 } variables & $\mathrm{F}_{1,468}$ & $\mathrm{~F}_{1,468}$ & $\mathrm{~F}_{1,468}$ & \\
BMI & 193.6 & 261.1 & 185.3 & 4 \\
Waist & 231.9 & 272.7 & 234.5 & 3 \\
WHR & 52.2 & 53.1 & 48.4 & 5 \\
WHTR & 268.5 & 323.8 & 260.4 & 2 \\
ABSI & 18.2 & 12.2 & 21.8 & 6 \\
WHT.5R & 278.3 & 333.5 & 275.8 & 1 \\
\hline
\end{tabular}


Table 4. The estimated parameter (B) obtained from the ANCOVA, predicting logtransformed sum-of-waist skinfolds Ln(SSK).

Dependent variable $\operatorname{Ln}(\mathrm{SSK})$

95\% Confidence Interval for

\begin{tabular}{llllll} 
Model & $\begin{array}{l}\text { Parameters } \\
(\mathrm{B})\end{array}$ & $\mathrm{SE}$ & $\mathrm{P}$ & $\begin{array}{l}\text { Lower } \\
\text { Bound }\end{array}$ & $\begin{array}{l}\text { Upper } \\
\text { Bound }\end{array}$ \\
\hline constant Ln(a) & .119 & 1.875 & .949 & -3.565 & 3.803 \\
Ln(W) & 3.751 & .200 & .000 & 3.358 & 4.143 \\
Ln(HT) & -2.340 & .383 & .000 & -3.093 & -1.588 \\
age & -.030 & .010 & .002 & -.049 & -.011 \\
age $^{2}$ & .0004 & .0001 & .0064 & .0001 & .0007 \\
male $(\Delta \operatorname{Ln}(\mathrm{a}))$ & -.426 & .058 & .000 & -.541 & -.311 \\
sport $(\Delta \operatorname{Ln}(\mathrm{a}))$ & -.037 & .052 & .478 & -.138 & .065 \\
male*sport $(\Delta \operatorname{Ln}(\mathrm{a}))$ & -.161 & .069 & .020 & -.297 & -.025 \\
\hline The female controls were used to estimate the baseline constant measure Ln(a) and all other
\end{tabular}

The female controls were used to estimate the
groups compared with it, indicated by $\Delta \operatorname{Ln}(\mathrm{a})$. 
Table 5. The estimated parameters obtained from fitting the non-linear least-squares regression model equation (3)

\begin{tabular}{lllll} 
Parameter & Estimate & Std. Error & $\begin{array}{l}\text { 95\% Confidence Interval } \\
\text { Lower Bound }\end{array}$ & $\begin{array}{l}\text { Upper } \\
\text { Bound }\end{array}$ \\
\hline b & -0.641 & 0.092 & -0.822 & -0.46 \\
constant a & 4.308 & 0.484 & 3.356 & 5.26 \\
male $(\Delta \mathrm{a})$ & -0.358 & 0.094 & -0.542 & -0.174 \\
male*sport $(\Delta \mathrm{a})$ & -0.059 & 0.013 & -0.084 & -0.034 \\
age & -0.007 & 0.002 & -0.011 & -0.002 \\
age & $8.77 \mathrm{E}-05$ & $3.50 \mathrm{E}-05$ & $1.99 \mathrm{E}-05$ & $1.56 \mathrm{E}-04$ \\
$\mathrm{~W}_{0}$ & 136.393 & 12.518 & 111.794 & 160.991 \\
$\mathrm{~W}_{0} *$ male $\left(\Delta \mathrm{W}_{0}\right)$ & -39.097 & 14.867 & -68.311 & -9.883
\end{tabular}

The female controls were used to estimate the baseline constant a, and all other groups compared with it, indicated by $\Delta \mathrm{a}$. 